\title{
Adult metastatic yolk sac tumor descending from an intra-abdominal testis: A case report and review of the literature
}

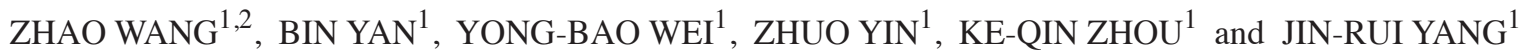 \\ ${ }^{1}$ Department of Urology, The Second Xiangya Hospital, Central South University, Changsha, Hunan 410011; \\ ${ }^{2}$ Department of Urology, Xiangya Hospital, Central South University, Changsha, Hunan 410008, P.R. China
}

Received November 12, 2014; Accepted September 18, 2015

DOI: $10.3892 / \mathrm{ol} .2015 .3817$

\begin{abstract}
Pure yolk sac tumors are extremely rare in adults; to the best of our knowledge, $<20$ cases have been reported. Multiple metastases originating from a pure yolk sac testicular tumor, descending from an intra-abdominal testis, are additionally extremely rare. In the present case, a man exhibiting a 30-year history of cryptorchidism and indirect inguinal hernia, was admitted to the Department of Urology (The Second Xiangya Hospital, Changsha, China) due to a mass that had descended from the abdominal cavity 7 months previously. Elevated levels of specific serum marker ( $\alpha$-fetoprotein, lactate dehydrogenase and human chorionic gonadotropin ) did not indicate potential testicular germ cell types prior to surgery and pathological examination. Pathological results and immunohistochemistry revealed a testicular pure yolk sac tumor subsequent to surgery. The present case report and literature review describes the typical characteristics of an adult testicular yolk sac tumor, as well as the diagnosis and management of the disease.
\end{abstract}

\section{Introduction}

In recent years, the incidence of testicular cancer has increased, particularly in industrialized countries (1). For example, in the USA, the incidence of testicular germ cell tumors increased from 5.7 to 6.8 cases per 100,000 individuals between 1992 and $2009(1,2)$. Testicular yolk sac tumors are a type of non-seminomatous germ cell tumor (NSGCT) $(1,3)$. Testicular pure yolk sac tumors are rare in adults (4). Cases of multiple metastases, originating from a testicular pure yolk sac tumor descending from a testis, which descended from the intra-abdominal cavity, are extremely rarely diagnosed (4). By performing a search of PubMed, it was identified that $<20$ cases of adult pure yolk

Correspondence to: Professor Jin-Rui Yang, Department of Urology, The Second Xiangya Hospital, Central South University, 139 Middle Renmin Road, Changsha, Hunan 410011, P.R. China E-mail: yjinrui@yahoo.com

Key words: yolk sac tumor, cryptorchidism, sperm cryopreservation, metastasis, chemotherapy sac tumor have been reported (4-11), and $<5$ cases of multiple metastasis have been reported $(5,10,11)$. Fludeoxyglucose F 18-positron emission tomography computed tomography is a useful tool to evaluate systematic conditions prior to further treatment. Males without offspring may choose to undergo sperm cryopreservation prior to chemotherapy. Follow-up and evaluation are necessary for advanced adult testicular cancer. The current study presents the case of a 30-year-old man, with a diagnosis of testicular pure yolk sac tumor accompanied by multiple metastases. Written informed consent was obtained from the patient's family. Relevant literature concerning incidence, diagnosis, treatment and prognosis of testicular pure yolk sac tumors are also reviewed. The current case report aims to increase knowledge with regard to this rare type of testicular cancer, which may improve diagnosis and treatment.

\section{Case report}

A 30-year-old man presented in September 2014 with a novel and progressively increasing swelling of the left scrotal contents for $\sim 7$ months. The patient was referred to the Department of Urology of The Second Xiangya Hospital (Changsha, China), following an ultrasound that indicated the presence of a testicular tumor.

The medical history of the patient was as follows: i) Cryptorchidism and indirect inguinal hernia in the left scrotum for 30 years; ii) secondary tuberculosis in 2005, and a directly observed treatment short-course for 6 months. The family history did not demonstrate anything notable.

Physical examination revealed an enlarged, hard and slightly tender mass in the left scrotal sac. No sinuses, ulcers or varicose veins were identified on the skin of the scrotum. A transillumination test was negative, indicating that the intra-scrotal mass was a tumor as opposed to a hydrocele.

Lactate dehydrogenase (LDH) measured $469.6 \mathrm{U} / 1$ (normal value, $<245 \mathrm{U} / \mathrm{l}) ; \beta$-human chorionic gonadotropin $(\beta$-hCG) was 103,541 $\mathrm{mIU} / \mathrm{ml}$ (normal value, $<2 \mathrm{mIU} / \mathrm{ml}$ ) and $\alpha$-fetoprotein (AFP) measured 6,252 ng/ml (normal value, $<5.8 \mathrm{ng} / \mathrm{ml}$ ). Tuberculin purified protein derivative (PPD)-immunoglobulin (Ig) G, PPD-IgM and MycoDot testing were positive. C-Reactive protein measured $20.10 \mathrm{mg} / \mathrm{l}$ (normal value, $<8 \mathrm{mg} / \mathrm{l}$ ) and erythrocyte sedimentation rate was $23 \mathrm{~mm} / \mathrm{h}$ (normal value, $<15 \mathrm{~mm} / \mathrm{h}$ ). A chest X-ray revealed pulmonary tuberculosis with fibrous bundles and calcification in the upper lung regions. An 


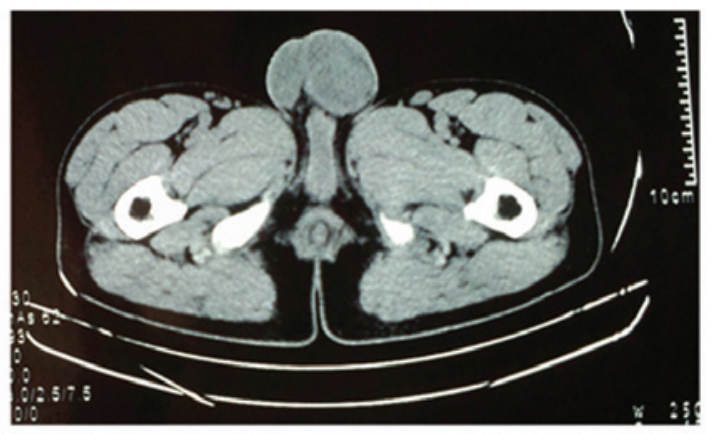

Figure 1. Preoperative imaging. Computed tomography scanning revealed a heterogeneous mass located in the left scrotum.

A

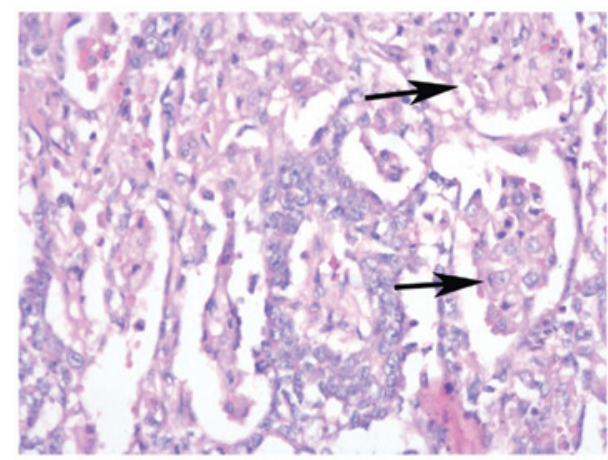

B

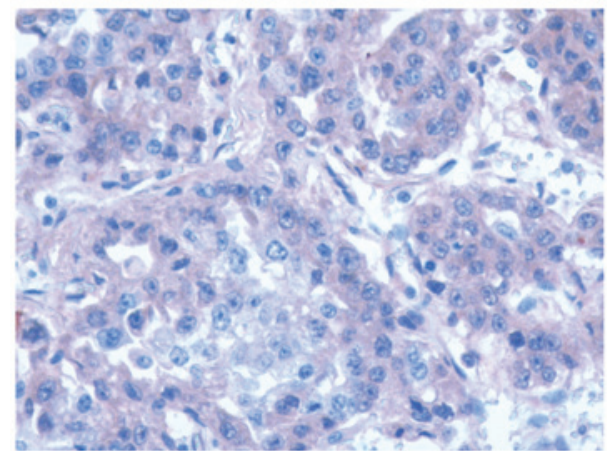

Figure 2. Stained tumor sections (A) Hematoxylin and eosin staining of the tumor (magnification, x200) revealed that the tumor cells were arranged in a reticular pattern with Schiller Duval bodies (black arrows); (B) Immunohistochemistry of the tumor was positive for $\alpha$-fetoprotein (magnification, x200).

ultrasound revealed a solid, heterogeneous mass, $\sim 40 \times 34 \mathrm{~mm}$ in the left scrotum. In addition, dot- and strip-like echo was detected in the mass, indicating poor vascular flow. Pelvic computed tomography (CT) scanning additionally revealed a heterogeneous mass characterized by ring-like shape and flake high density (Fig. 1).

In order to diagnose the scrotal mass, which had descended from the abdominal cavity, left inguinal exploration and radical orchiectomy was performed. The excised specimen exhibited a hard, red surface, and some necrosis and hemorrhaging were observed upon sectioning. Pathological results indicated that the specimen was a germ cell tumor. Subsequent immunohistochemistry revealed AFP (+), hCG (-), creatine kinase (+), Ki-67 (30\%+), octamer-binding transcription factor 3/4 (-) and placental alkaline phosphatase (partly+). The final pathological diagnosis reached was a testicular pure yolk sac tumor (Fig. 2).
A

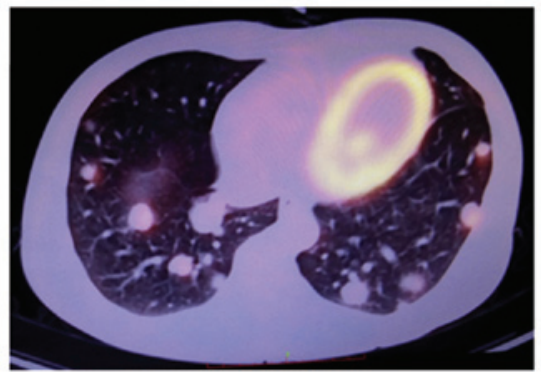

B

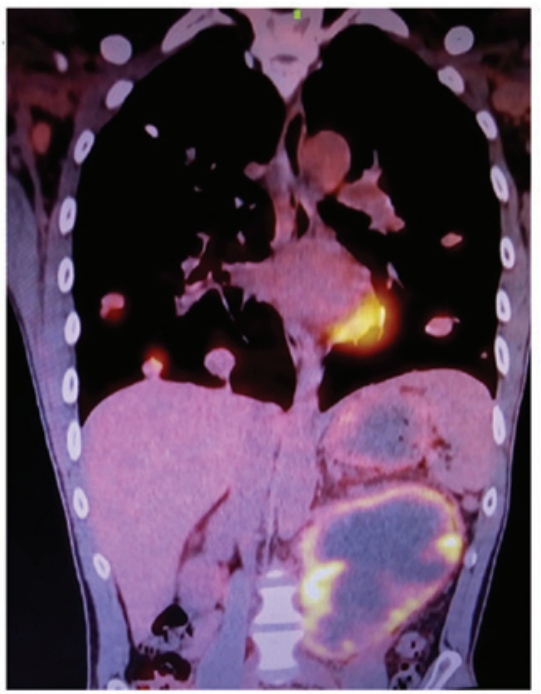

Figure 3. Postoperative imaging. (A) Fludeoxyglucose F 18-positron emission tomography (FDG-PET) scanning revealed multiple soft tissue masses exhibiting increased glucose metabolism bilaterally in the lungs. (B) Abdominal masses exhibiting ring-like increased glucose metabolism in an FDG-PET scan.

Prior to advanced treatment, fludeoxyglucose F 18 (FDG)-positron emission tomography (PET) assessment was performed in order to evaluate the general condition of the patient and the presence of any distal metastases. FDG-PET indicated the presence of lung and inguinal lymph node metastases, as well as a mass in the abdominal cavity (Fig. 3). The patient was advised to undergo semen cryopreservation, and subsequent cisplatin, etoposide and bleomycin (PEB) combination chemotherapy regimen $\left(20 \mathrm{mg} / \mathrm{m}^{2}\right.$ cisplatin, days $1-5$; $100 \mathrm{mg} / \mathrm{m}^{2}$ etoposide, days $1-5 ; 30 \mathrm{mg}$ bleomycin, days 1 , 8 and 15). However, the patient and his family have currently rejected the advice, and the patient did not receive any further therapy.

\section{Discussion}

A testicular yolk sac tumor is a type of NSGCT $(1,3)$. Testicular yolk sac tumors are common in children exhibiting testicular cancer, however, they are rarely observed in adults (4). According to a global epidemiological investigation, the incidence of testicular cancer has been increasing in a number of countries between the 1980s and the 2000s; however, the overall mortality rate has remained low between the 1970 s and the 2000s (12). The same survey additionally revealed that New Zealand, Australia and Sweden exhibited the three highest age-standard incidence rates of testicular cancer globally; China demonstrated one of the lowest mortality rates for 
testicular cancer (12). However, the annual percentage increase in testicular cancer incidence has been observed to be $3.5 \%$ in China (12). In a Japanese report (13), seminoma accounted for $80.8 \%$ of testicular cancer cases; by contrast yolk sac tumor cases accounted for $18.0 \%$ in 2005 and 2008, respectively (mean age of patients, 37.0 \pm 10.6 ). PubMed was searched using the keywords 'adult testicular cancer' or 'yolk sac tumor'. The results indicated that $<20$ adult pure yolk sac tumors have been reported (4-11). Furthermore, multiple organ metastases associated with yolk sac tumors are even rarer $(5,10,11)$.

Cryptorchidism is a significant risk factor for testicular cancer $(14,15)$. In the present case, the patient had possessed a history of cryptorchidism for the previous 30 years. The present study hypothesized that carcinogenesis occurred prior to the retained testis descending from the abdominal cavity. The patient palpated a hard mass and detected a bulge in the left scrotum 7 months prior to hospital admittance. Indirect inguinal hernia may have altered the local structure of the inguinal canal,forming a passage that allowed the intra-abdominal testis to descend. Alternatively, repeated descending and recurrence of the hernia may have caused inflammation of the inguinal canal and intra-abdominal testes. Thus, adhesion may form between the hernia and testis, causing the hernia sac to pull the intra-abdominal testis down as it descended from the abdominal cavity.

LDH, $\beta$-hCG and AFP are the recommended serum markers for the evaluation of testicular cancer (1). AFP is not elevated in seminoma; however, AFP elevation is detectable in 40-60\% advanced NSGCT cases (16). hCG may be elevated in all types of NSGCT (16). In the present case, all three serum markers were elevated, particularly AFP and hCG. This was useful as it is difficult for urologists to evaluate the pathological patterns of NSGCTs prior to surgery. In the present case, pathological analysis did not detect trophoblastic cells, and hCG was negative in immunohistochemical testing in numerous sample sections. According to Ulbright et al (17), the immunohistochemistry results were consistent with a yolk sac tumor. A total of 7 days following orchiectomy, significant levels of LDH, $\beta$-hCG and AFP were detected, similar to the levels measured prior to surgery. The elevated hCG levels may not have been due to the testicular cancer; a coexisting tumor may have been causing the persistent elevation (16). An FDG-PET procedure was proposed by the treating urologists in order to evaluate the general condition of the patient, as well as the presence of metastases or coexisting tumors. A potential abdominal malignancy may have been a factor causing the significant positivity of hCG. LDH, $\beta$-hCG and AFP serum markers are generally effective tools for the evaluation of risk stratifications and treatment decisions, however, combination analysis and general evaluation may additionally be necessary $(16,18)$.

Spermatogenesis and semen quality are altered following chemotherapy for the treatment of testicular cancer, and the degree of alteration is associated with the type and intensity of chemotherapy $(19,20)$. According to a survey conducted by Molnar et al (19), sperm concentration is reduced in men exhibiting testicular tumors compared with matched healthy men. In a French national investigation, sperm concentration and total sperm number were significantly decreased in NSGCT patients following orchiectomy, therefore sperm cryopreservation should be considered prior to orchiectomy (21). In a number of retrospective studies (22-24), sperm cryopreservation has been recommended for testicular cancer patients who intend to have a child in the future. The man in the present case had been married for 2 years, and intended to have a child in the future, therefore sperm cryopreservation may be an option.

According to testicular cancer guidelines $(1,3,25)$, the current patient presented with a stage III tumor, therefore a PEB chemotherapy regimen was proposed for treatment. Follow-up assessments including general condition, serum markers and PET-CT should be regularly performed following chemotherapy. In a Japanese multicenter study (13), the prognosis of testicular cancer was positive; 1-year and 3-year overall survival rates were 98.3 and $96.8 \%$, respectively. European and USA data indicated that the 5-year relative survival rate of NSGCT patients varied from $90-95 \%$ in $15-29$ - and 30-49-year olds (26). However, in the case of the patient in the current study, due to the presence of multiple metastases and an abdominal tumor, regular follow-up visits remained necessary in order to evaluate long-term survival.

In the present case of multiple metastases originating from a pure testicular yolk sac tumor, serum markers and FDG-PET may assist with the achievement of a differential diagnosis. Sperm cryopreservation is necessary for adults who intend to have a child in the future. Chemotherapy is recommended in order for patients to achieve tumor remission. In terms of ongoing evaluation of the remission of metastases, serum marker evaluation may provide an indicator of patient prognosis.

\section{Acknowledgements}

The authors would like to thank Dr Daiqiang Li and Dr Xiaoling She in the Department of Pathology (The Second Xiangya Hospital, Changsha, China), for their assistance with the analysis of pathological results.

\section{References}

1. Albers P, Albrecht W, Algaba F, et al: Guidelines on Testicular Cancer: 2015 Update. Eur Urol: Aug 18, 2015 (Epub ahead of print).

2. Nigam M, Aschebrook-Kilfoy B, Shikanov S and Eggener S: Increasing incidence of testicular cancer in the United States and Europe between 1992 and 2009. World J Urol 33: 623-631, 2015.

3. Vasdev N, Moon A and Thorpe AC: Classification, epidemiology and therapies for testicular germ cell tumours. Int J Dev Biol 57: 133-139, 2013.

4. Khan S, Jetley S, Pujani M and Neogi S: Pure yolk sac tumor of testis in an adult: A rare occurrence. J Postgrad Med 60: 351-353, 2014.

5. Balzer BL and Ulbright TM: Spontaneous regression of testicular germ cell tumors: An analysis of 42 cases. Am J Surg Pathol 30: 858-865, 2006.

6. Medica M, Germinale F, Giglio M, Stubinski R, Campodonico F, Raggio $M$ and Carmignani G: Adult testicular pure yolk sac tumor. Urol Int 67: 94-96, 2001.

7. Foster RS, Hermans B, Bihrle R and Donohue JP: Clinical stage I pure yolk sac tumor of the testis in adults has different clinical behavior than juvenile yolk sac tumor. J Urol 164: 1943-1944, 2000.

8. Munver R, Kronz JD and Polascik TJ: HIV infection presenting as an unusually large pure yolk sac tumor of the testis. J Urol 164: 1653-1654, 2000.

9. Montserrat Orri V, López-Bonet E, Garijo G, et al: Pure yolk sack tumor of the testis in adults: Report of a case. Actas Urol Esp 20: 659-661, 1996. 
10. Hashimoto Y, Iwase Y, Mogami T, Hayashi Y, Sasaki S, Kato M, Tugaya $\mathrm{M}$ and Kohri K: A case of adult pure yolk sac tumor of the testis achieving pathological complete response by chemotherapy. Hinyokika Kiyo 41: 813-816, 1995 (In Japanese).

11. Izumi H, Shiokawa H, Shibata Y, Kurokawa J and Ohbu M: Pure yolk sac tumor of the testis with brain metastasis: Report of an adult case. Hinyokika Kiyo 38: 1071-1074, 1992 (In Japanese).

12. Shanmugalingam T, Soultati A, Chowdhury S, Rudman S and Van Hemelrijck M: Global incidence and outcome of testicular cancer. Clin Epidemiol 5: 417-427, 2013.

13. Miki T, Kamoi K, Fujimoto H, Kanayama HO, Ohyama C, Suzuki K, Nishiyama H, Eto M, Naito S, Fukumori T, et al: Clinical characteristics and oncological outcomes of testicular cancer patients registered in 2005 and 2008: The first large-scale study from the Cancer Registration Committee of the Japanese Urological Association. Int J Urol 21: S1-S6, 2014.

14. Cook MB, Akre O, Forman D, Madigan MP, Richiardi L and McGlynn KA: A systematic review and meta-analysis of perinatal variables in relation to the risk of testicular cancer - experiences of the son. Int J Epidemiol 39: 1605-1618, 2010.

15. Pinczowski D, McLaughlin JK, Läckgren G, Adami HO and Persson I: Occurrence of testicular cancer in patients operated on for cryptorchidism and inguinal hernia. J Urol 146: 1291-1294, 1991.

16. Gilligan TD, Seidenfeld J, Basch EM, Einhorn LH, Fancher T, Smith DC, Stephenson AJ, Vaughn DJ, Cosby R and Hayes DF; American Society of Clinical Oncology: American Society of Clinical Oncology Clinical Practice Guideline on uses of serum tumor markers in adult males with germ cell tumors. J Clin Oncol 28: 3388-3404, 2010

17. Ulbright TM, Tickoo SK, Berney DM and Srigley JR; Members of the ISUP Immunohistochemistry in Diagnostic Urologic Pathology Group: Best practices recommendations in the application of immunohistochemistry in testicular tumors: Report from the International Society of Urological Pathology consensus conference. Am J Surg Pathol 38: e50-e59, 2014.

18. Ruf CG, Sachs S, Khalili-Harbi N, Isbarn H, Wagner W, Matthies C, Meineke V, Fisch M, Chun FK and Abend M: Prediction of metastatic status in non-seminomatous testicular cancer. World J Urol 32: 1205-1211, 2014.
19. Molnar Z, Mokanszki A, Kassai Bazsane Z, Bhattoa HP, Benyo M, Olah E and Jakab A: Sperm concentration, hyaluronic acid-binding capacity, aneuploidy and persistent histones in testicular cancer. Hum Reprod 29: 1866-1874, 2014.

20. Paoli D, Gallo M, Rizzo F, et al: Testicular cancer and sperm DNA damage: Short-and long-term effects of antineoplastic treatment. Andrology 3: 122-128, 2015.

21. Rives N, Perdrix A, Hennebicq S, Saïas-Magnan J, Melin MC, Berthaut I, Barthélémy C, Daudin M, Szerman E, Bresson JL, et al: The semen quality of 1158 men with testicular cancer at the time of cryopreservation: Results of the French National CECOS Network. J Androl 33: 1394-1401, 2012.

22. Chung JP, Haines CJ and Kong GW: Sperm cryopreservation for Chinese male cancer patients: A 17-year retrospective analysis in an assisted reproductive unit in Hong Kong. Hong Kong Med J 19: 525-530, 2013.

23. Molnár Z, Berta E, Benyó M, Póka R, Kassai Z, Flaskó T, Jakab $A$ and Bodor M: Fertility of testicular cancer patients after anticancer treatment - experience of 11 years. Pharmazie 69: 437-441, 2014.

24. Záková J, Lousová E, Ventruba P, Crha I, Pochopová H, Vinklárková J, Tesařová E and Nussir M: Sperm cryopreservation before testicular cancer treatment and its subsequent utilization for the treatment of infertility. ScientificWorldJournal 2014: 575978, 2014.

25. Oldenburg J, Fosså SD, Nuver J, Heidenreich A, Schmoll HJ, Bokemeyer C, Horwich A, Beyer J, Kataja V; ESMO Guidelines Working Group: Testicular seminoma and non-seminoma: ESMO Clinical Practice Guidelines for diagnosis, treatment and follow-up. Ann Oncol 24 (Suppl 6): vi125-vi132, 2013.

26. Verhoeven RH, Gondos A, Janssen-Heijnen ML, Saum KU, Brewster DH, Holleczek B, Crocetti E, Rosso S, Hakulinen T, Aareleid T and Brenner H: Testicular cancer in Europe and the USA: Survival still rising among older patients. Ann Oncol 24: 508-513, 2013 